\title{
Optic nerve head injury and optical coherence tomography angiography
}

\author{
Xiaoli Ma, Rui Hua \\ Department of Ophthalmology, First Hospital of China Medical University, Shenyang, China \\ Correspondence to: Rui Hua. Department of Ophthalmology, The First Affiliated Hospital of China Medical University, No. 155 Nanjingbei Street, \\ Heping District, Shenyang, China. Email: woodshua@126.com.
}

Submitted Oct 31, 2020. Accepted for publication Apr 09, 2021.

doi: 10.21037/qims-20-1218

View this article at: http://dx.doi.org/10.21037/qims-20-1218

Dear editors,

We wrote this letter to discuss the microvascular abnormalities related to optic nerve head $(\mathrm{ONH})$ injury visualized by optical coherence tomography angiography (OCTA). With the advent of OCTA, different fundus vasculature levels in the macular region can be displayed precisely and noninvasively to investigate its mechanisms. OCTA is a promising diagnostic approach for detecting inflammatory, ischemic, and compressive optic neuropathies (1). However, the potential relationship between parapapillary microvascular dysfunction and $\mathrm{ONH}$ injury is still unclear and should be investigated by combined structural and functional OCT.

The macula and the ONH's different microvasculature results in a weaker association of the vascular density (VD) of the macula with tissue thickness compared to $\mathrm{ONH}$ VD (2). Anatomically, the optic disc's surface (i.e., the superficial nerve fiber layer) is supplied by superficial ONH capillaries derived from the central retinal artery (3). The retinal ganglion cell axons in the peripapillary nerve fiber layer are fed by distinct capillary networks originating from adjacent retinal arterioles (4). It has been reported that both global and sectoral circumpapillary VD on the $\mathrm{ONH}$ surface, excluding the papillomacular bundle area, decreases significantly with age (5). Moreover, the anterior lamina cribrosa is supplied by short posterior ciliary arteries, the Zinn-Haller arterial circle, and partially by the peripapillary choroidal vasculature $(3,6)$. Finally, the posterior lamina cribrosa is supplied through various sources, mainly the peripheral pia arteries and short posterior ciliary arteries of the Zinn-Haller arterial circle (3).

Peripapillary OCTA vessel parameters have greater intrasession repeatability than intersession reproducibility in both non-glaucomatous and glaucomatous eyes (7). Previous studies have compared the flow pattern using OCTA in the peripapillary region in normal eyes, glaucomatous eyes, and acute nonarteritic anterior ischemic optic neuropathy (NAION) eyes. Normal subjects showed VD's steepest slopes in the nasal superior and inferior circumpapillary sectors (-0.098\%/year and $-0.096 \% /$ year, respectively) (5). Conversely, in patients with NAION, OCTA revealed decreased peripapillary VD and peripapillary vascular dilatation and tortuosity (8). Similarly, patients with papilledema have dilated and tortuous capillaries at the ONH surface (9).

In contrast, glaucoma-related decreased peripapillary retinal perfusion can be detected as a focal defect pattern using OCTA (10). ONH perfusion detected by optical microangiography showed significant differences between glaucomatous eyes and normal controls and was significantly correlated with disease severity and structural defects in glaucomatous eyes (11). Furthermore, glaucomatous eyes with focal lamina cribrosa defects are usually accompanied by peripapillary microvasculature dropout (12). Our clinical practice has identified blood flow signals in the deep anterior lamina cribrosa via both spectral-domain OCTA and swept-source OCTA (Figures 1,2), providing a new biomarker for assessing the blood supply of the optic disc. Numa et al. reported blood flow signals visualized on or immediately adjacent to lamina beams, outside of lamina pores, using en face OCTA (13). 


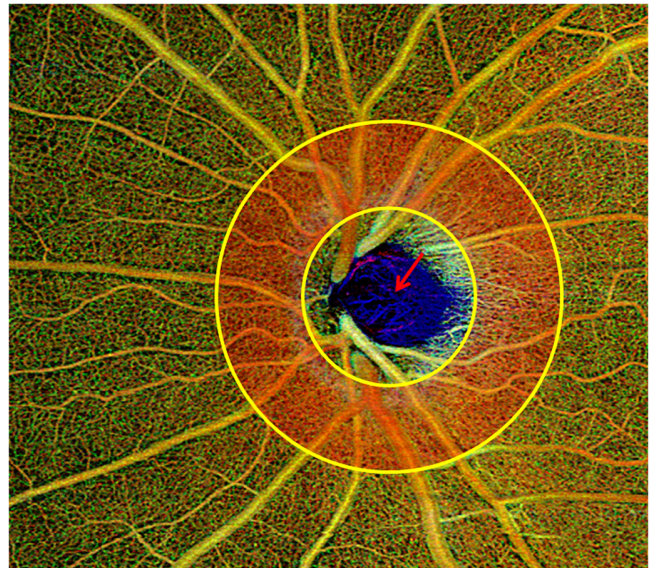

Figure 1 Swept-source OCTA (Zeiss elite 9000) images of the $\mathrm{ONH}$ include both the circumpapillary region (red area) and the anterior lamina cribrosa (red arrow). OCTA, optical coherence tomography angiography; ONH, optic nerve head.
Glaucoma is a chronic progressive optic nerve degeneration caused by high intraocular pressure (IOP), resulting in injury and death of retinal ganglion cells and their axons and visual field deficits. OCTA can assess the structural and functional progression of glaucoma (14). Usually, circumpapillary retinal nerve fiber layer (cpRNFL) thinning is accompanied by flow voids in the peripapillary region on OCTA. In contrast to normal eyes, patients with glaucoma have lower peripapillary flow index and VD values, accounting for the corresponding pattern standard deviation in the visual field test (10). Retinal nerve fiber layer (RNFL) blood flow metrics show significant correlations with visual field indices and structural changes in glaucomatous eyes (15). Chen et al. also detected reduced RNFL microcirculation in the normal hemisphere of eyes with glaucoma, with strong correspondence with VF loss and RNFL thinning (16). In addition to superficial vascular
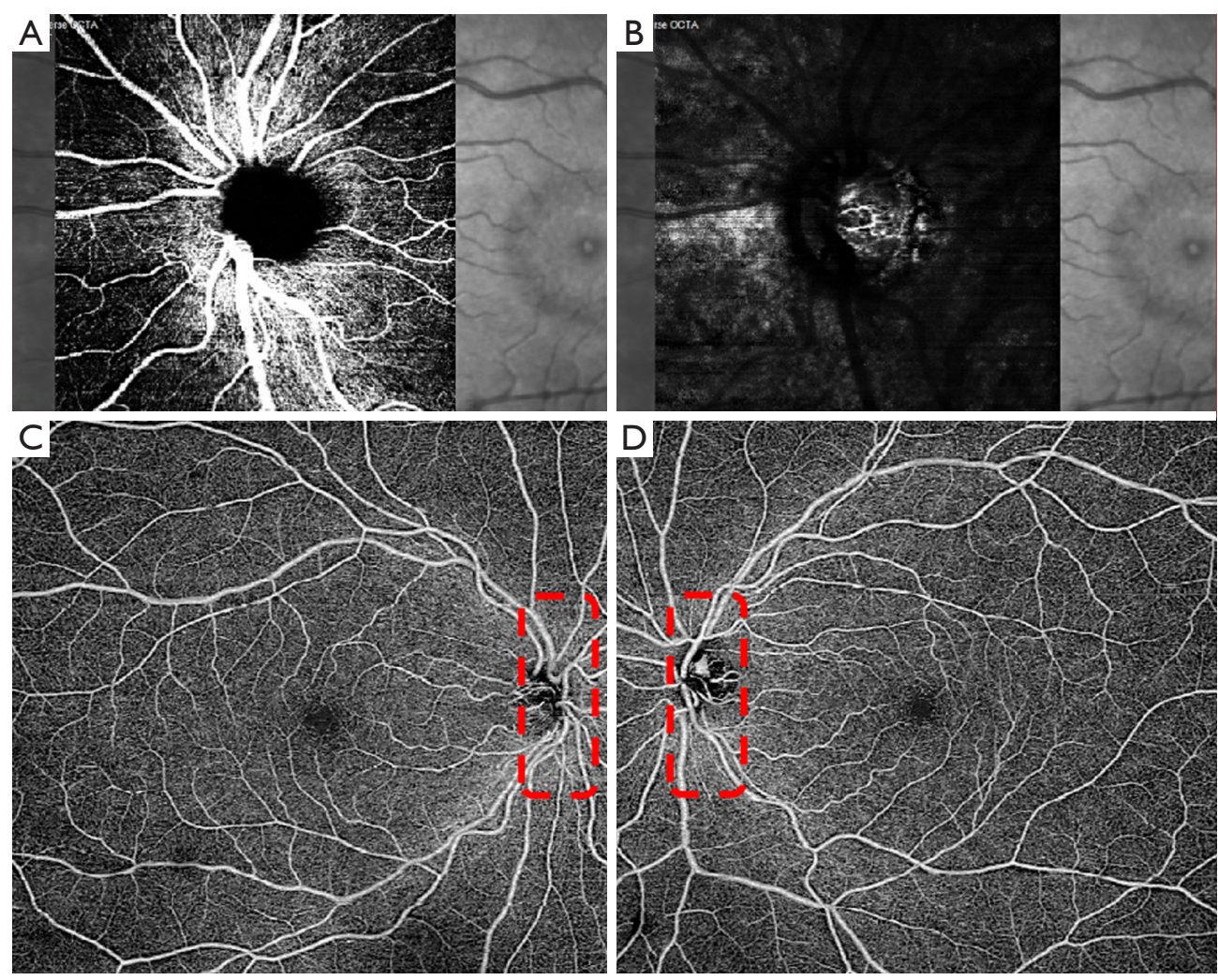

Figure 2 OCTA images of the anterior lamina cribrosa. (A) Spectral-domain OCTA (Heidelberg-Engineering; Spectralis) showing the parapapillary microvascular system in the left eye. (B) Spectral-domain OCTA (Heidelberg-Engineering; Spectralis) revealing the microvasculature of the anterior lamina cribrosa in the left eye after changing the segmentation (the same patient as in A). (C,D) Sweptsource OCTA (Zeiss elite 9000) showing the microvascular system in both the parapapillary region and the anterior lamina cribrosa (red dots rectangles) binocularly. OCTA, optical coherence tomography angiography. 

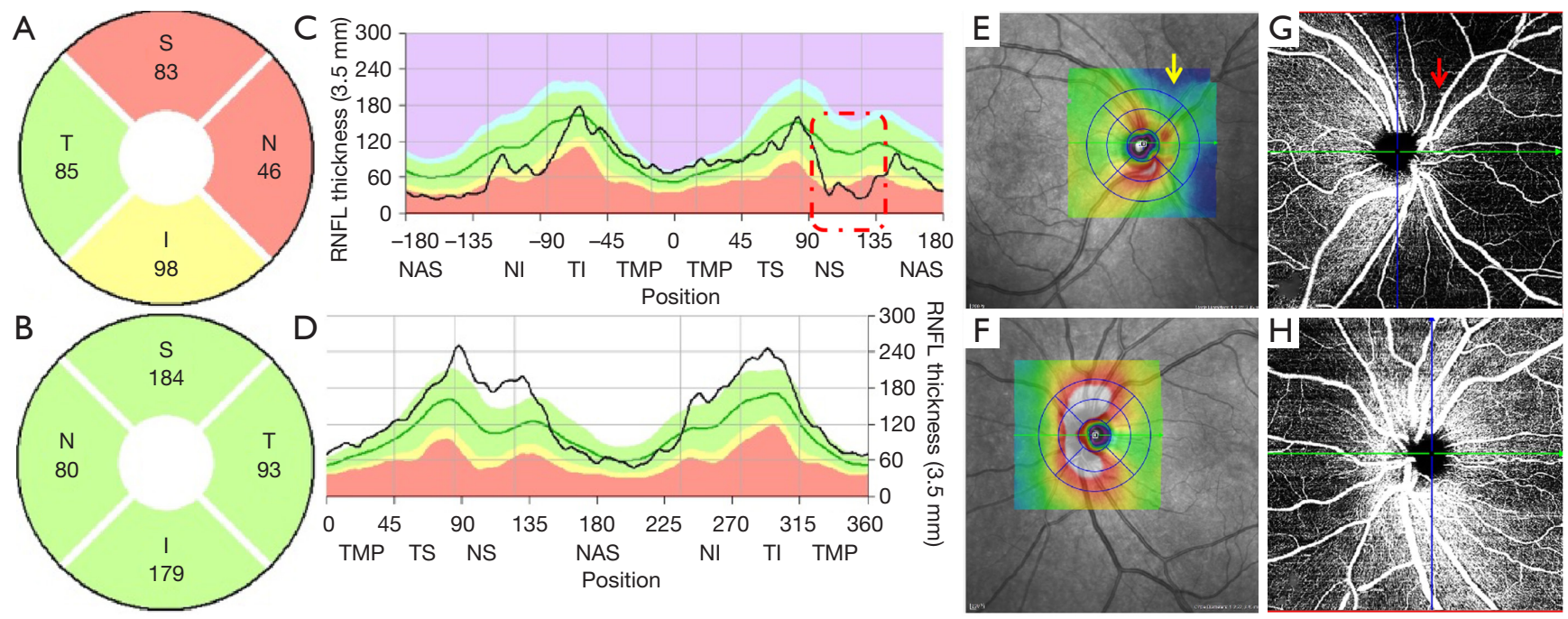

Figure 3 Correlation between retinal structural abnormalities and the corresponding blood flow in a glaucoma case. Compared with the left eye (B), the parapapillary nerve fiber is atrophic in superior and nasal sections (A); correspondingly, the RNFL is thinning in the right eye (C, red dots rectangle), but the RNFL thickness is normal in the left eye (D); the three dimensional OCT (Heidelberg-Engineering; Spectralis) image of the $\mathrm{ONH}$ in the right eye visualized local depression (E, yellow arrow), which is in accordance with the flow void region (red arrow) in en face OCTA $(\mathrm{G})$; in contrast, both ONH topography (F) and en face OCTA $(\mathrm{H})$ are normal in the left eye. OCTA, optical coherence tomography angiography; ONH, optic nerve head; RNFL, retinal nerve fiber layer.

plexuses, glaucoma affects the deep vascular plexuses, initially leading to axonal injury (14). There is an association between parapapillary deep-layer microvasculature dropout and corresponding focal reductions in juxtapapillary choroidal thickness in the parapapillary $\gamma$-zone of primary open-angle glaucomatous eyes (17). Interestingly, we found good correspondence between cpRNFL values, ONH topography, and en face OCTA, suggesting capillary atrophy involvement in glaucomatous progression (Figure 3).

Manalastas et al. reported a strong relationship between ONH VD and both cpRNFL and macular ganglion cell complex (GCC) thickness (2). Aside from significantly thinner cpRNFL and its lower circumpapillary VD, glaucomatous eyes with microvasculature dropout show lower IOP, larger cup-to-disc ratio, and worse visual field mean deviation in comparison to glaucomatous eyes with no microvasculature dropout (18). In contrast to normal eyes, glaucomatous eyes usually have a sectoral reduction in lamina cribrosa microvasculature blood flow (13).

Late-stage NAION has a specific nerve atrophy pattern, including sectional cpRNFL thinning and tempo-superior or tempo-inferior successive dark blankets on posterior pole asymmetry analysis (PPAA, Figure 4). Glaucoma and localized RNFL defects were successfully detected using
PPAA by evaluating the difference between the upper and lower macular thickness (19).

In a previous study, OCTA parameters showed stronger associations with functional rather than structural measures of glaucoma, and glaucomatous eyes initially had reduced macular ganglion cell-inner plexiform layer microcirculation (20). Optic disc injury is commonly associated with macular GCC shrinkage, which is related to lower $\mathrm{ONH}$ perfusion (21). Moreover, parapapillary microvascular dysfunction has been reported in NAION cases. For example, compared to normal eyes, eyes with NAION showed a lower average VD in the superior $(42.1 \% \pm 2.4 \%)$, nasal $(46.1 \% \pm 3.3 \%)$, inferior $(43.7 \% \pm 1.8 \%)$, and temporal $(47.6 \% \pm 2.9 \%)$ quadrants $(8)$.

Optic disc melanocytoma (ODM), a pigmented tumor, appears as a brownish-black focal lesion with feathery margins. On blue-light fundus autofluorescence (FAF), it appears as enlarged hypo-autofluorescence in the ODM region (22). Tumor vascularization, manifested by fluorescein leakages, strongly indicates the growth of ODM (23). Moreover, the laser speckle flowgraph showed reduced blood flow in the ODM region, indicating that ODM induces ONH circulatory disorder and leads to a specific visual field defect (22). 

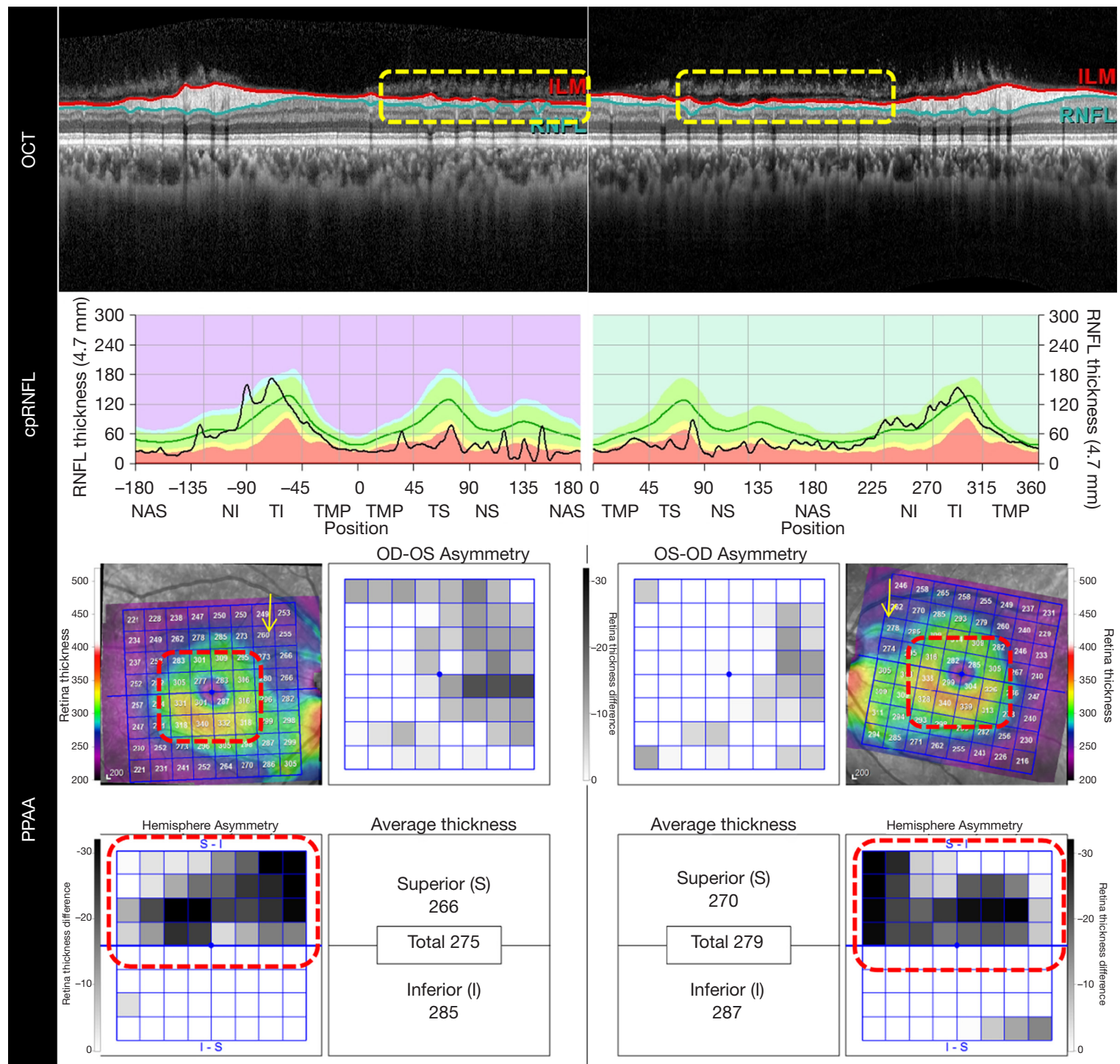

Right eye

Figure 4 Sectional optic nerve atrophy of a bilateral NAION case in the late stage: OCT (Heidelberg-Engineering; Spectralis) shows sectional parapapillary nerve fiber atrophy bilaterally (yellow dots rectangles); Correspondingly, the cpRNFL reveals parapapillary nerve fiber thinning in tempo-superior and tempo-nasal sections in both eyes; PPAA demonstrates tempo-superior depression (yellow arrows), superior half atrophy of the GCC (red dots rectangles in macular regions), and tempo-superior absolute successive dark blankets in the posterior pole (red dots rectangles in the analytic maps). cpRNFL, circumpapillary retinal nerve fiber layer; GCC, ganglion cell complex; NAION, nonarteritic anterior ischemic optic neuropathy; OCT, optical coherence tomography; PPAA, posterior pole asymmetry analysis.

In our clinical experience, ODM has a normal appearance on multicolor imaging. However, on melanin-related near infrared (NIR)-FAF, confirmed by enhanced depth imaging OCT, ODM appears as typical hyper-fluorescence, which may indicate the final diagnosis (Figure 5). NIR-FAF reflects melanin's metabolism in the retinal pigment epithelium and choroids at a longer wavelength (24).

OCTA can visualize the superficial fine abnormal retinal vessels on the ODM surface, also indicating tumor growth. However, Carnevali et al. reported that OCTA detected 

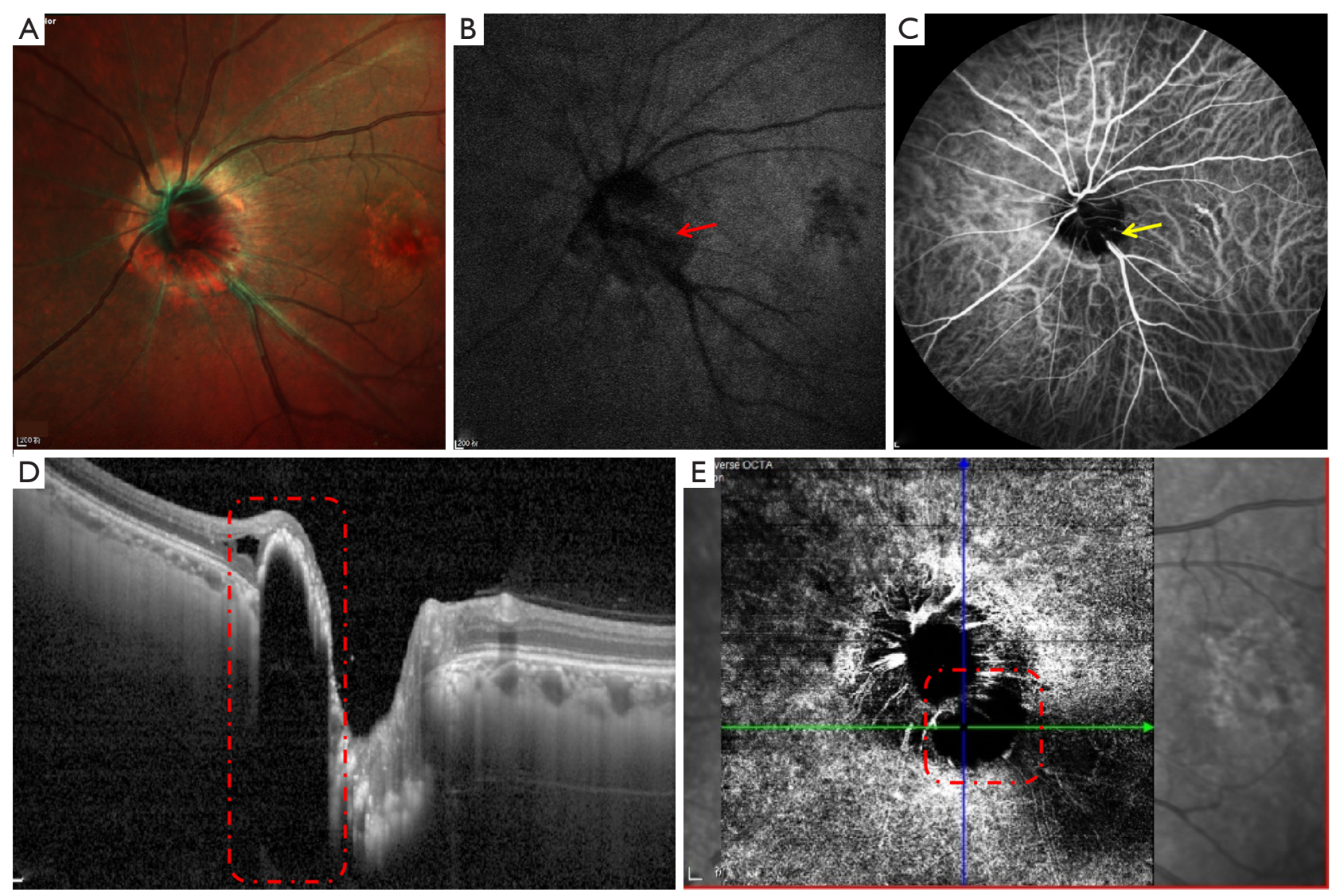

Figure 5 Multimodal imaging of an ODM in the left eye. (A) Multicolor imaging showing normal outlook of the optic disc and patched atrophy in the macula; (B) NIR-FAF showing typical focal hyper-fluorescence adjacent to the optic disc (red arrow). (C) Indocyanine green angiography absent in intratumoral vessels (yellow arrow). (D) Enhanced depth imaging OCT (Heidelberg-Engineering; Spectralis) visualizing a parapapillary hyper-reflective lesion (red dots rectangle), with nasal retinoschisis. (E) The segmentation of the intratumoral level not showing any blood flow signal. The green arrow indicates the orientation of OCT in illustration D. NIR-FAF, near infrared fundus autofluorescence; OCT, optical coherence tomography; ODM, optic disc melanocytoma.

superficial tumor vascularization in 2 out of 5 eyes (22). Conversely, no intratumoral vessels were found using neither indocyanine green angiography nor en face OCTA in our study. Similarly, Cennamo et al. reported 2 eyes with ODM demonstrating flow voids and defects in the deep retinal layer (25). These typical OCTA findings may indicate an inactive stage of ODM.

OCTA is crucial for better understanding ONH injury lesions' pathogenesis because it can show different changes in the microvascular ONH abnormalities (both optic disc and peripapillary region) at different levels. As OCTA technology continues to advance at an accelerated pace, we expect to discover new perspectives in the domain of glaucoma and neuro-ophthalmology to optimize the diagnostic sensitivity and specificity.

\section{Acknowledgments}

Funding: This work was supported by the Fund for Clinical Genetics (Ophthalmology) Subject construction project of China Medical University (No. 3110118049), Natural Science Foundation of Liaoning Province (No. 20170541041), Beijing Bethune Charitable Foundation (No. AF-OG-03-1.1-03).

\section{Footnote}

Conflicts of Interest: Both authors have completed the 
ICMJE uniform disclosure form (available at http://dx.doi. org/10.21037/qims-20-1218). The authors have no conflicts of interest to declare.

Ethical Statement: The Institutional Review Board of the First Hospital of China Medical University approved this study, and informed consent was obtained from all individual participants.

Open Access Statement: This is an Open Access article distributed in accordance with the Creative Commons Attribution-NonCommercial-NoDerivs 4.0 International License (CC BY-NC-ND 4.0), which permits the noncommercial replication and distribution of the article with the strict proviso that no changes or edits are made and the original work is properly cited (including links to both the formal publication through the relevant DOI and the license). See: https://creativecommons.org/licenses/by-nc-nd/4.0/.

\section{References}

1. Chwalisz BK, Bouffard MA, Prasad S, Cestari DM. Neuroimaging diagnostic and monitoring approaches in ophthalmology. Curr Opin Neurol 2018;31:66-73.

2. Manalastas PIC, Zangwill LM, Daga FB, Christopher MA, Saunders LJ, Shoji T, Akagi T, Penteado RC, Yarmohammadi A, Suh MH, Medeiros FA, Weinreb RN. The Association Between Macula and ONH Optical Coherence Tomography Angiography (OCT-A) Vessel Densities in Glaucoma, Glaucoma Suspect, and Healthy Eyes. J Glaucoma 2018;27:227-32.

3. Onda E, Cioffi GA, Bacon DR, Van Buskirk EM. Microvasculature of the human optic nerve. Am J Ophthalmol 1995;120:92-102.

4. Yarmohammadi A, Zangwill LM, Diniz-Filho A, Suh MH, Manalastas PI, Fatehee N, Yousefi S, Belghith A, Saunders LJ, Medeiros FA, Huang D, Weinreb RN. Optical Coherence Tomography Angiography Vessel Density in Healthy, Glaucoma Suspect, and Glaucoma Eyes. Invest Ophthalmol Vis Sci 2016;57:OCT451-9.

5. Jo YH, Sung KR, Shin JW. Effects of Age on Peripapillary and Macular Vessel Density Determined Using Optical Coherence Tomography Angiography in Healthy Eyes. Invest Ophthalmol Vis Sci 2019;60:3492-8.

6. Na KI, Lee WJ, Kim YK, Jeoung JW, Park KH. Evaluation of Optic Nerve Head and Peripapillary Choroidal Vasculature Using Swept-source Optical Coherence Tomography Angiography. J Glaucoma
2017;26:665-8.

7. Lee JC, Grisafe DJ, Burkemper B, Chang BR, Zhou $\mathrm{X}$, Chu Z, Fard A, Durbin M, Wong BJ, Song BJ, Xu BY, Wang R, Richter GM. Intra-session Repeatability and Inter-session Reproducibility of Peripapillary OCTA Vessel Parameters in Non-Glaucomatous and Glaucomatous Eyes. Br J Ophthalmol 2020. [Epub ahead of print]. doi: 10.1136/bjophthalmol-2020-317181.

8. Al-Nashar HY, Hemeda S. Assessment of peripapillary vessel density in acute non-arteritic anterior ischemic optic neuropathy. Int Ophthalmol 2020;40:1269-76.

9. Rougier MB, Le Goff M, Korobelnik JF. Optical coherence tomography angiography at the acute phase of optic disc edema. Eye Vis (Lond) 2018;5:15.

10. Liu L, Jia Y, Takusagawa HL, Pechauer AD, Edmunds B, Lombardi L, Davis E, Morrison JC, Huang D. Optical Coherence Tomography Angiography of the Peripapillary Retina in Glaucoma. JAMA Ophthalmol 2015;133:1045-52.

11. Chen CL, Bojikian KD, Gupta D, Wen JC, Zhang Q, Xin C, Kono R, Mudumbai RC, Johnstone MA, Chen PP, Wang RK. Optic disc perfusion in normal eyes and eyes with glaucoma using optical coherence tomography-based microangiography. Quant Imaging Med Surg 2016;6:125-33.

12. Suh MH, Zangwill LM, Manalastas PI, Belghith A, Yarmohammadi A, Medeiros FA, Diniz-Filho A, Saunders LJ, Yousefi S, Weinreb RN. Optical Coherence Tomography Angiography Vessel Density in Glaucomatous Eyes with Focal Lamina Cribrosa Defects. Ophthalmology 2016;123:2309-17.

13. Numa S, Akagi T, Uji A, Suda K, Nakanishi H, Kameda T, Ikeda HO, Tsujikawa A. Visualization of the Lamina Cribrosa Microvasculature in Normal and Glaucomatous Eyes: A Swept-source Optical Coherence Tomography Angiography Study. J Glaucoma 2018;27:1032-35.

14. Mwanza JC, Budenz DL. New developments in optical coherence tomography imaging for glaucoma. Curr Opin Ophthalmol 2018;29:121-9.

15. Chen CL, Zhang A, Bojikian KD, Wen JC, Zhang Q, Xin C, Mudumbai RC, Johnstone MA, Chen PP, Wang RK. Peripapillary Retinal Nerve Fiber Layer Vascular Microcirculation in Glaucoma Using Optical Coherence Tomography-Based Microangiography. Invest Ophthalmol Vis Sci 2016;57:OCT475-85.

16. Chen CL, Bojikian KD, Wen JC, Zhang Q, Xin C, Mudumbai RC, Johnstone MA, Chen PP, Wang RK. Peripapillary Retinal Nerve Fiber Layer Vascular Microcirculation in Eyes With Glaucomaand Single- 
Hemifield Visual Field Loss. JAMA Ophthalmol 2017;135:461-8.

17. Lee SH, Lee EJ, Kim TW. Topographic correlation between juxtapapillary choroidal thickness and parapapillary deep-layer microvasculature dropout in primary open-angle glaucoma. Br J Ophthalmol 2018;102:1134-40.

18. Akagi T, Zangwill LM, Shoji T, Suh MH, Saunders LJ, Yarmohammadi A, Manalastas PIC, Penteado RC, Weinreb RN. Optic disc microvasculature dropout in primary open-angle glaucoma measured with optical coherence tomography angiography. PLoS One 2018;13:e0201729.

19. Hua R, Gangwani R, Guo L, McGhee S, Ma X, Li J, Yao K. Detection of preperimetric glaucoma using Bruch membrane opening, neural canal and posterior pole asymmetry analysis of optical coherence tomography. Sci Rep 2016;6:21743.

20. Richter GM, Madi I, Chu Z, Burkemper B, Chang R, Zaman A, Sylvester B, Reznik A, Kashani A, Wang RK, Varma R. Structural and functional associations of macular microcirculation in the ganglion cell-inner plexiform layer in glaucoma using optical coherence tomography

Cite this article as: Ma X, Hua R. Optic nerve head injury and optical coherence tomography angiography. Quant Imaging Med Surg 2021;11(10):4497-4503. doi: 10.21037/qims-20-1218 angiography. J Glaucoma 2018;27:281-90.

21. Wang X, Jiang C, Ko T, Kong X, Yu X, Min W, Shi G, Sun X. Correlation between optic disc perfusion and glaucomatous severity in patients with openangle glaucoma: an optical coherence tomography angiography study. Graefes Arch Clin Exp Ophthalmol 2015;253:1557-64.

22. Carnevali A, Querques L, Zucchiatti I, Scorcia V, Bandello F, Querques G. Optical Coherence Tomography Angiography Features in Melanocytoma of the Optic Nerve. Ophthalmic Surg Lasers Imaging Retina 2017;48:364-6.

23. Lee CS, Bae JH, Jeon IH, Byeon SH, Koh HJ, Lee SC. Melanocytoma of the optic disk in the Korean population. Retina 2010;30:1714-20.

24. Hua R, Yao K, Liu L, Li J. Near infrared autofluorescence findings in diffuse subretinal fibrosis syndrome. Lasers Med Sci 2015;30:2395-7.

25. Cennamo G, Romano MR, Breve MA, Velotti N, Reibaldi M, de Crecchio G, Cennamo G. Evaluation of choroidal tumors with optical coherence tomography: enhanced depth imaging and OCT-angiography features. Eye (Lond) 2017;31:906-15. 